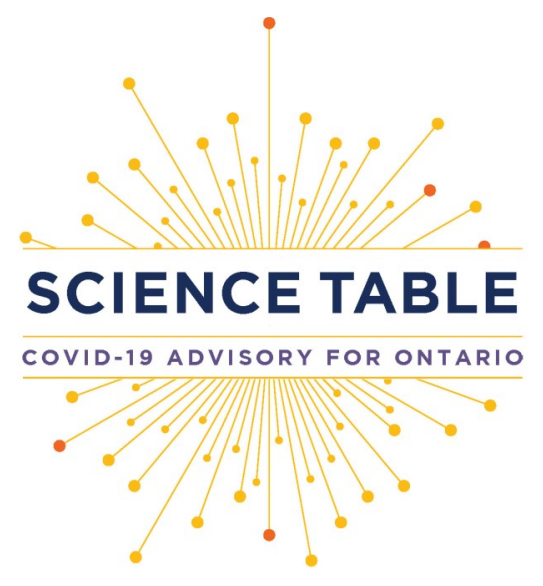

Version 1.0

Published: March 31, 2021

Citation: Stall NM, Nakamachi Y, Chang M, et al. Mobile in-home COVID-19 vaccination of Ontario homebound older adults by neighbourhood risk. Science Briefs of the Ontario COVID-19 Science Advisory Table. 2021;1(19). $\quad$ https://doi.org/10.47326/ ocsat.2021.02.19.1.0

Author Affiliations: The affiliations of the members of the Ontario COVID-19 Science Advisory Table can be found at https:// covid19-sciencetable.ca/.

Declarations of Interest: The declarations of interest of the members of the Ontario COVID-19 Science Advisory Table, its Working Groups, or its partners can be found at https://covid19-sciencetable.ca/. The declarations of interest of external authors can be found under additional resources at https:// doi.org/10.47326/ocsat.2021.02.19.1.0.

About Us: The Ontario COVID-19 Science Advisory Table is a group of scientific experts and health system leaders who evaluate and report on emerging evidence relevant to the COVID-19 pandemic, to inform Ontario's response. Our mandate is to provide weekly summaries of relevant scientific evidence for the COVID-19 Health Coordination Table of the Province of Ontario, integrating information from existing scientific tables, Ontario's universities and agencies, and the best global evidence. The Science Table summarizes its findings for the Health Coordination Table and the public in Science Briefs.

Correspondence to: Secretariat of the Ontario COVID-19 Science Advisory Table (info@covid19-sciencetable.ca)

Copyright: 2021 Ontario COVID-19 Science Advisory Table. This is an open access document distributed under the terms of the Creative Commons Attribution License, which permits unrestricted use, distribution, and reproduction in any medium, provided that the original work is properly cited.

The views and findings expressed in this Science Brief are those of the authors and do not necessarily reflect the views of all of the members of the Ontario COVID-19 Science Advisory Table and its partners.

\title{
Mobile In-Home COVID-19 Vaccination of Ontario Homebound Older Adults by Neighbourhood Risk
}

Nathan M. Stall, Yoshiko Nakamachi, Melissa Chang, Shiran Isaacksz, Christa Sinclair Mills, Elizabeth Niedra, Camille Lemieux, Kevin A. Brown, Andrew P. Costa, Brian Hodges, Tai Huynh, Sophia Ikura, Aaron Jones, Lauren Lapointe-Shaw, Antonina Maltsev, Allison McGeer, Paula A. Rochon, Arjumand Siddiqi, Adam Thurston, Peter Jüni, Samir K. Sinha on behalf of the Ontario COVID-19 Science Advisory Table

\section{Key Message}

Homebound individuals face substantial barriers to receiving COVID-19 vaccines as they cannot or rarely leave their homes because of medical, psychiatric, cognitive, functional, transportation-related and social reasons.

There are at least 75,000 Ontarians aged 65 years and above who are homebound, with the majority being women and people aged 85 years and above. Much of this older homebound population requires mobile in-home COVID-19 vaccination, which could be prioritized by residence in high SARS-CoV-2 risk neighbourhoods.

\section{Summary}

\section{Background}

As the Ontario COVID-19 vaccination program continues to roll out, equitable distribution strategies are essential for those who face barriers to vaccination. One population facing such barriers are homebound adults aged 65 years and above.

As of March 29, 2021, 303,552 or 26.4\% of Ontarians aged 75 years and above had neither received one dose of a COVID-19 vaccine nor booked an appointment to receive one, with mass vaccination clinic bookings for that age group declining.

\section{Questions}

How many Ontario older adult long-stay home care recipients are homebound?

What are the sociodemographic characteristics of homebound older adults, including residence in high SARS-CoV-2 incidence neighbourhoods?

What are the key logistical considerations for mobile in-home COVID-19 vaccination of homebound older adults?

\section{Findings}

Between January 1 and December 31, 2020, there were 76,755 long-stay recipients of publicly funded home care services in Ontario aged 65 years and above who were homebound ( $1.1 \%$ of the Ontario population aged 65 years and above).

Identifying all homebound older Ontarians, over and above those receiving publicly funded homecare services, requires leveraging provincial and regional databases, community- and home-based primary care providers, community support services, neighbourhood ambassadors, and community outreach workers. 
Key logistical considerations for mobile in-home vaccination of homebound older adults involve mobilizing and training healthcare professionals and non-clinical staff, ensuring that there is a sufficient number of vaccinators in the field who are wellcoordinated, appropriately planning the number of vaccine doses needed, mapping out travel plans, and monitoring and maintaining vaccine temperature during transportation.

\section{Interpretation}

Many homebound individuals are currently or will soon be prioritized in Ontario's COVID-19 vaccination program. With nearly 113,139 or $16.7 \%$ of all Ontarians aged 80 years and above having neither received a COVID-19 vaccine nor booked an appointment to receive a vaccine, it is imperative that mobile in-home vaccination outreach is available to the homebound population.

\section{Background}

As the Ontario COVID-19 vaccination program continues to roll out, equitable distribution strategies are essential for those who face barriers to vaccination. ${ }^{1}$ One population facing such barriers are homebound adults aged 65 years and above. ${ }^{2}$ The homebound population is defined as community-dwelling individuals who cannot or rarely leave their homes because of medical, psychiatric, cognitive, functional, transportation-related or social reasons. ${ }^{3,4}$ Homebound individuals are also at increased risk of SARS-CoV-2 infections transmitted by visitors and home care workers, and COVID-19 hospitalizations and deaths due to their underlying risk factors.

As of March 29, 2021, 303,552 or 26.4\% of Ontarians aged 75 years and above had neither received one dose of a COVID-19 vaccine nor booked an appointment to receive one, with bookings for that age group declining. ${ }^{5}$ With the overwhelming majority of older Canadians expressing an intent to receive the COVID-19 vaccine, there may be a substantial proportion of older Ontarians who want to get vaccinated but are unable to attend a vaccination clinic. ${ }^{6}$

\section{Questions}

How many Ontario older adult long-stay home care recipients are homebound?

What are the sociodemographic characteristics of homebound older adults, including residence in high SARS-CoV-2 incidence neighbourhoods?

What are the key logistical considerations for mobile in-home COVID-19 vaccination of homebound older adults?

\section{Findings}

Between January 1 and December 31,2020, there were 76,755 long-stay home care recipients aged 65 years and above who were homebound $(1.1 \%$ of the Ontario population aged 65 years and above). Of these homebound older adults, 49,714 (64.8\%) were women, and 41,304 (53.8\%) were aged 85 years and above, 23,884 (31.1\%) were aged 75 to 84 years, and 11,567 (15.1\%) were aged 65 to 74 years. In Toronto and Peel, the majority of homebound older adults lived in the top 20\% of neighbourhoods with the highest cumulative SARS-CoV-2 incidence between January 23, 2020 and January 16, 2021 (Figure 1). ${ }^{7}$

There are several key logistical considerations when vaccinating homebound older adults. ${ }^{8}$ Homebound Ontarians requiring mobile in-home COVID-19 vaccination should first be identified through their primary care providers and publicly-funded 
long-stay home care programs and by leveraging a number of additional channels. Homebound individuals and their caregivers should be able to indicate their requirement for in-home vaccination on provincial and public health nit (PHU) vaccine booking systems or through other mechanisms. Homebound individuals who may have difficulty navigating these systems should be further identified and supported to sign up for vaccination through their primary care providers, home care providers and community support services programs like Meals on Wheels, and by neighbourhood ambassadors and community outreach workers. ${ }^{9}$

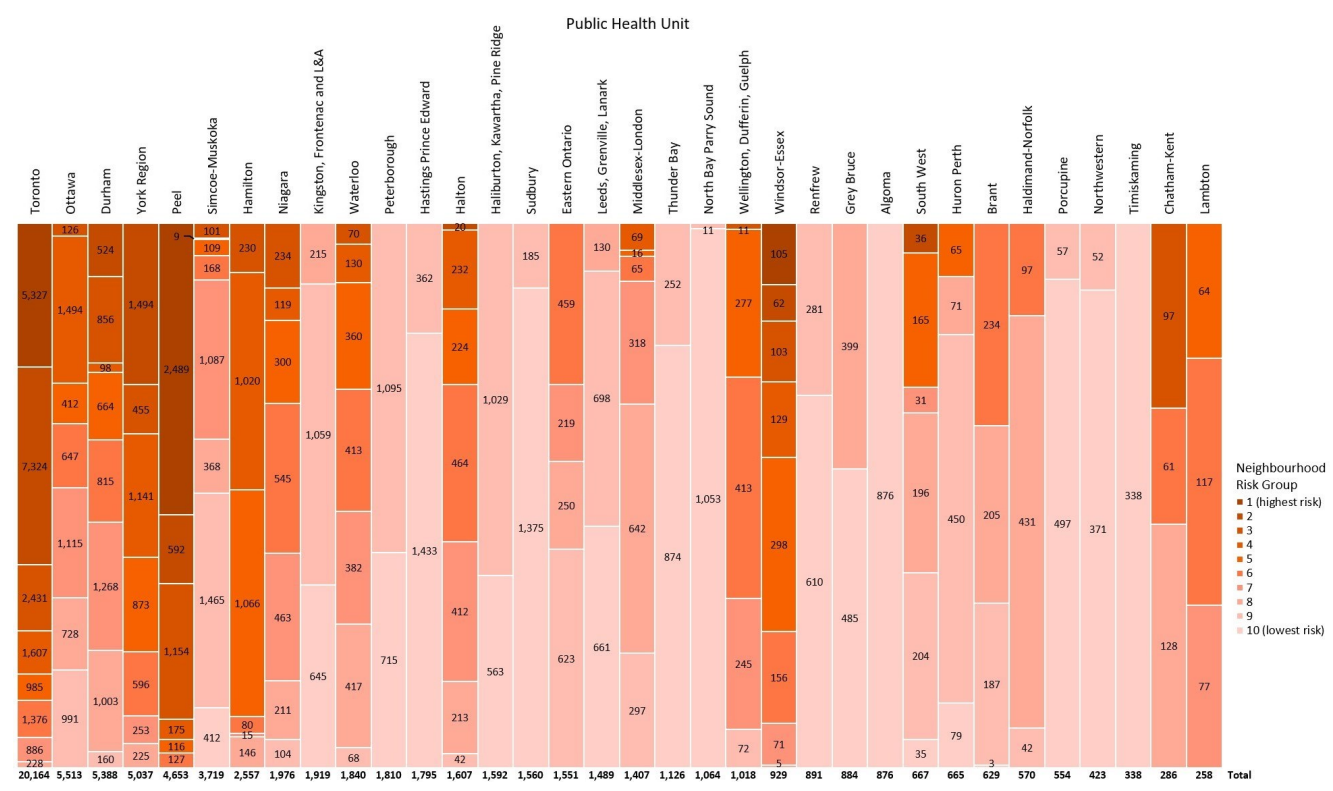

Figure 1. Number of Ontario Long-Stay Home Care Recipients Aged 65 or Older Who Were Homebound in 2020, by Public Health Unit and Neighbourhood Incidence of SARS-CoV-2 Infection

Table describing the number of Ontario long-stay home care recipients aged 65 or older who were homebound in 2020. Home care clients who have or are expected to have care needs extending beyond 60 days are classified as long-stay. Homebound individuals were identified using the Resident Assessment Instrument - Home Care (RAI-HC) in the calendar year 2020. Homebound older adults were sorted by residence in one of Ontario's 34 public health units (PHUs) and further by residence in a neighbourhood ranked in $10 \%$ increments by cumulative incidence of SARS -CoV-2 infection between Jan 23, 2020 and Jan 16, 2021. Neighbourhoods are defined by the first three characters of a resident's postal code, known as "forward sortation area". Group 1 includes neighbourhoods with the highest cumulative incidence of SARS-CoV-2 infection, whereas group 10 includes neighbourhoods with the lowest cumulative incidence of SARS-CoV-2 infection. Data for cumulative incidence of SARS-CoV-2 infections sourced from the Public Health Case and Contact Management Solution and other case management systems (CCM plus), extracted on January 16, 2021; data for homebound long-stay home care recipients sourced from the Ontario Home Care Database (HCD), January 1 to December 31, 2020.

Many community support services use standardized assessments that are similar to those used in home care, which can be used to identify clients who are homebound. Paramedic services, particularly those that operate community paramedicine programs, can further identify older adults that are homebound. Wherever possible, trusted relationships should be leveraged to address any COVID-19 vaccine related questions or concerns and provide education.

The Ontario Ministry of Health has developed guidance documents for the onward transport of COVID-19 vaccines beyond the initial point of delivery and interim guidance for the transport of syringes containing COVID-19 vaccine for exceptional circumstances, including for the vaccination of homebound clients. ${ }^{10}$ In-home COVID -19 vaccination efforts should involve mobilizing and training healthcare professionals and non-clinical staff to support mobile clinics, which could include primary care providers, home care and public health practitioners, and community paramedics. ${ }^{11}$ Given the sizeable population of homebound older adults in Ontario and the laborious nature of mobile in-home COVID-19 vaccination, it will be crucial to ensure that there is a sufficient number of vaccinators in the field, and that they 
are well-coordinated to minimize inefficiencies and duplications.

Mobile in-home COVID-19 vaccination is also resource intensive. Rapidly vaccinating the homebound older adult population will require leveraging existing client and care provider relationships while building the capacity of clinicians within the health system. As an example, the City of Toronto is rolling out three homebound vaccination models in partnership with Ontario Health Toronto Region, regional home care program leads for the City of Toronto, Toronto Paramedic Services, the University Health Network, and local primary care teams in partnership with Toronto Public Health (Figure 2).

\begin{tabular}{|c|c|c|}
\hline Model 1 & Model 2 & Model 3 \\
\hline Led by primary care teams & $\begin{array}{l}\text { Collaboration between home care, } \\
\text { home-based primary care or community } \\
\text { service providers with a Toronto } \\
\text { Paramedic Services or a } \\
\text { primary care team }\end{array}$ & Led by Toronto Paramedic Services \\
\hline $\begin{array}{l}\text { Homebound individuals with primary } \\
\text { care providers who are able to provide } \\
\text { home-based vaccinations will have their } \\
\text { vaccination coordinated and completed } \\
\text { by a primary care team. } \\
\text { OHTs can be an integral part of ensuring } \\
\text { communication between primary care } \\
\text { providers regarding the identification of } \\
\text { homebound adults. Primary care } \\
\text { providers can be empowered to organize } \\
\text { local clinic or OHT-based programs to } \\
\text { vaccinate their regional patients. } \\
\text { Operationally, vaccination of homebound } \\
\text { adults through primary care providers } \\
\text { benefits from the support of an anchor } \\
\text { institution (e.g., a hospital or PHU) which } \\
\text { can provide access to vaccine supply, } \\
\text { support for safe handling of vaccines, and } \\
\text { logistical advice for operational issues } \\
\text { such consent and cOVax. }\end{array}$ & $\begin{array}{l}\text { Homebound individuals with an existing } \\
\text { relationship to home care, home-based } \\
\text { primary care, or community service } \\
\text { providers will have their vaccination } \\
\text { coordinated and completed by their } \\
\text { providers in collaboration with Toronto } \\
\text { Paramedic Services or primary care } \\
\text { teams. }\end{array}$ & $\begin{array}{l}\text { Homebound individuals who have no } \\
\text { providers who are able to provide home- } \\
\text { based vaccination will be supported by } \\
\text { the Toronto Paramedic Services to } \\
\text { complete their vaccination. } \\
\text { Individuals who do not currently receive } \\
\text { support from a home care provider or } \\
\text { home-visiting primary care provider, can } \\
\text { be referred for vaccination by their } \\
\text { primary care provider. } \\
\text { Unattached individuals, including those } \\
\text { without a primary care provider, may } \\
\text { reach out to Toronto Paramedic Services } \\
\text { and city support lines to be directed to } \\
\text { their Ontario Health region and linked to } \\
\text { the Senior Help Hotline. }\end{array}$ \\
\hline
\end{tabular}

Figure 2. Homebound COVID-19 Vaccination Models to be Rolled Out in the City of Toronto OHT, Ontario Health Team. PHU, Public Health Unit. In Ontario, COVax refers to COVaxON.

Mobile in-home COVID-19 vaccination also requires appropriate planning and logistical support to accurately estimate the number of doses needed, map out travel plans, and ensure the monitoring and maintenance of vaccine temperature during transportation. A sample map of the pre-vaccination, vaccination, post-vaccination and second dose stages of the mobile in-home COVID-19 vaccination process is detailed in Figure 3.

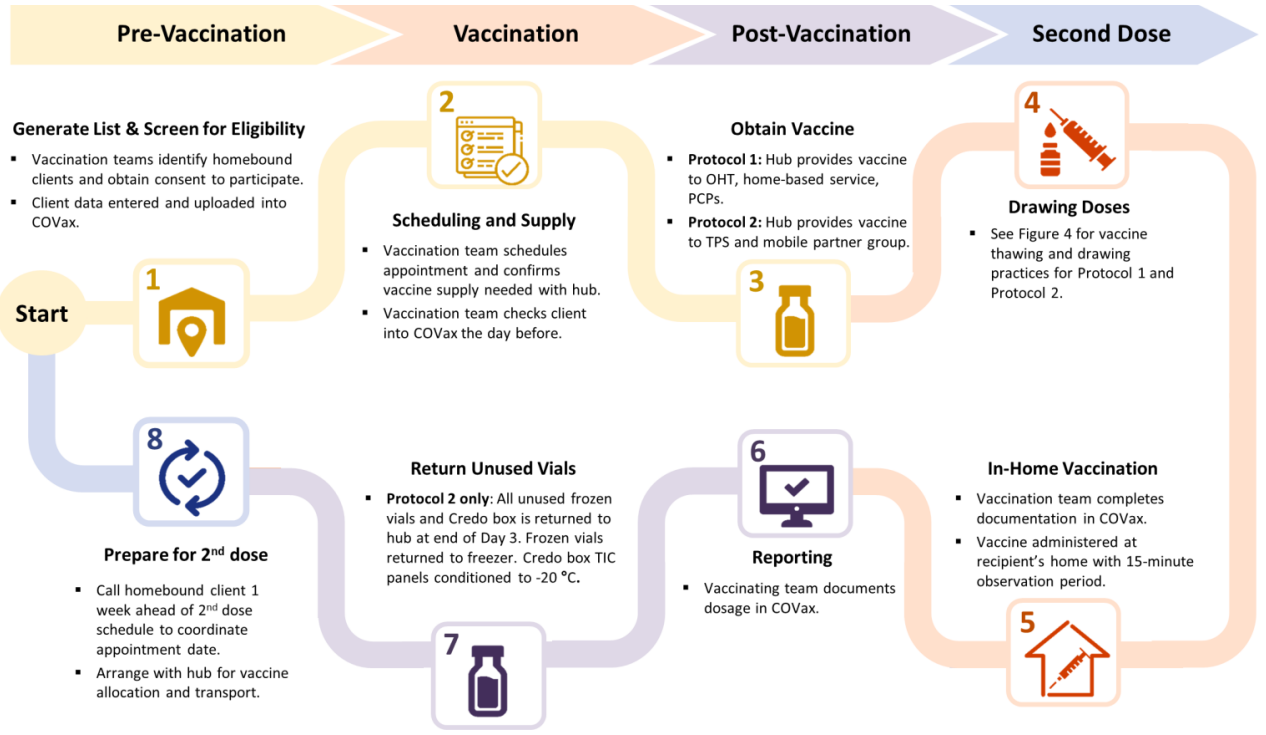

Figure 3. Map of Sample Mobile In-Home COVID-19 Vaccination Process for Homebound Individuals in Ontario Process being rolled out for the City of Toronto, which can be used as a sample mobile in-home COVID-19 vaccination 
process for homebound individuals across the Province of Ontario. Vaccination teams for Protocol 1 include OHTs, home-based services, and primary care providers. Vaccination teams for Protocol 2 include Toronto Paramedic Services and mobile partner groups. OHT, Ontario Health Team. PCP, primary care provider. TPS, Toronto Paramedic Services. TIC, thermal isolation chamber.

The mobile in-home COVID-19 vaccination protocol developed for the City of Toronto uses the Moderna mRNA vaccine, which has specific storage, transportation and handling requirements. ${ }^{12,13}$ The Moderna vaccine comes in a 10-dose vial and is stored in freezers between $-25^{\circ} \mathrm{C}$ to $-15^{\circ} \mathrm{C}$. To maintain the stability of the vaccine, the vials should only be transported in a frozen state. The thawed vaccine can be stored in refrigerators between $2^{\circ}$ to $8^{\circ} \mathrm{C}$ for up to 30 days prior to first use and cannot be refrozen. Unpunctured vials may be stored between $8^{\circ}$ to $25^{\circ} \mathrm{C}$ for up to 12 hours, and once a 10-dose vial is punctured, it must be used within 6 hours. While thawed vials should not be transported, thawed Moderna vaccine drawn up into syringes can be transported in UV protected sleeves for exceptional circumstances, such as vaccinating homebound individuals. Current Ontario Ministry of Health guidance documents maintain that a punctured or diluted vial or syringe with Pfizer-BioNTech COVID-19 vaccine should not be transported. ${ }^{10}$

\begin{tabular}{|c|c|}
\hline $\begin{array}{l}\text { Protocol } 1 \\
\text { Vaccine vials shipped from hub to vaccination team }\end{array}$ & $\begin{array}{c}\text { Protocol } 2 \\
\text { Syringes drawn and transported in thawed state }\end{array}$ \\
\hline $\begin{array}{l}\text { - Vaccination teams include } \mathrm{OHTs} \text {, home-based services, and PCPs. } \\
\text { team in preconditioned cooler set to }+2^{\circ} \mathrm{C} \text { to }+8^{\circ} \mathrm{C} \text {, with temperature } \\
\text { loggers with remote monitoring capabilities. } \\
\text { - Upon receipt, vaccine is stored in fridge for a maximum of } 30 \text { days. } \\
\text { - As needed, thawed vial is punctured, and vaccine is drawn into } \\
\text { syringes. All vaccine doses are to be drawn all at once and placed in } \\
\text { UV protective sleeves. Vaccines administered in syringes within } 6 \\
\text { hours from time of first vial puncture. } \\
\text { - Syringes distributed to other partner teams as needed. } \\
\text { - Syringes transported to homebound clients in preconditioned cooler } \\
\text { set to }+2^{\circ} \mathrm{C} \text { to }+8^{\circ} \mathrm{C} \text {, with temperature loggers with remote monitoring } \\
\text { capabilities. } \\
\text { - Homebound individuals are vaccinated in geographic clusters to } \\
\text { ensure vaccine is administered before the } 6 \text {-hour expiration time. }\end{array}$ & $\begin{array}{l}\text { - Vaccination teams include TPS and mobile partner groups. } \\
\text { - Frozen vials removed from hub freezer and transported in } \\
\text { remote monitoring capabilities. } \\
\text { - Credo box acts as a mobile freezer and can travel with mobile } \\
\text { vaccination team. } \\
\text { - As needed, frozen vial is removed from }-20^{\circ} \mathrm{C} \text { Credo box in time for } \\
\text { vaccine administration. } \\
\text { - Vaccine thawed in }+2^{\circ} \mathrm{C} \text { to }+8^{\circ} \mathrm{C} \text { cooler before drawing into syringes. } \\
\text { - Vaccine drawn into syringes with UV protective sleeves and } \\
\text { transported to vaccination team in a preconditioned cooler set to } \\
+2^{\circ} \mathrm{C} \text { to }+8^{\circ} \mathrm{C} \text {, with temperature loggers with remote monitoring } \\
\text { capabilities, as in Protocol } 1 \text {. Syringes distributed to other partner } \\
\text { teams as needed. } \\
\text { - Homebound individuals are vaccinated in geographic clusters to } \\
\text { ensure vaccine is administered before the } 6 \text {-hour expiration time. } \\
\text { - Mobile vaccinations teams have the flexibility to act as a mobile } \\
\text { syringe hub and can vaccinate several clusters consecutively when } \\
\text { traveling with vaccines in a Credo box. } \\
\text { - Frozen vials can remain in }-20^{\circ} \mathrm{C} \text { Credo box for a maximum of } 3 \text { days, } \\
\text { at which point they must be returned to the hub freezer or be stored } \\
\text { in a }+2^{\circ} \mathrm{C} \text { to }+8^{\circ} \mathrm{C} \text { fridge for } 30 \text { days, as in Protocol } 1 \text {. Only vaccines } \\
\text { that are still in a frozen state and have been in a Credo box the entire } \\
\text { time can be sent back to a hub freezer for storage. }\end{array}$ \\
\hline
\end{tabular}

Figure 4. Sample Mobile In-Home COVID-19 Vaccination Protocols for Homebound Individuals in Ontario Protocols being rolled out for the City of Toronto, which can be used as sample protocols for the Province of Ontario. The mobile in-home vaccination protocols are aligned with the Ontario Ministry of Health guidance documents for the onward transport of COVID-19 vaccines beyond the initial point of delivery and the interim guidance for transport of syringes containing COVID-19 vaccine for exceptional circumstances, including vaccination of homebound individuals. ${ }^{10}$ *The hub freezer can either precondition Credo box TIC panels in $-80^{\circ} \mathrm{C}$ freezers or arrange for delivery of already preconditioned $-20^{\circ} \mathrm{C}$ Credo boxes. OHT, Ontario Health Team. PCP, primary care providers. TIC, thermal isolation chamber.

In accordance with these storage, transportation, and handling requirements, two protocols have been developed for the vaccination of homebound individuals in the City of Toronto with the Moderna COVID-19 vaccine. Protocol 1 involves drawing up thawed vaccine into syringes at the site of freezing or refrigeration, and then directly transporting the vaccine in syringes in UV protected sleeves for mobile in-home vaccination within 6 hours. Protocol 2 involves transporting the vaccine in frozen vials using specialized reusable temperature-controlled parcel shippers (Credo boxes) and temperature loggers with remote monitoring capability. ${ }^{14}$ This provides the vaccination teams with the ability to thaw individual vials in the field for mobile in-home vaccination within 6 hours. Any unused vials can be returned to the hub freezer within 3 days if they are still in a frozen state and have been in a Credo box 
the entire time. These two protocols are detailed in Figures 4 and 5 and can be replicated across the Province of Ontario.

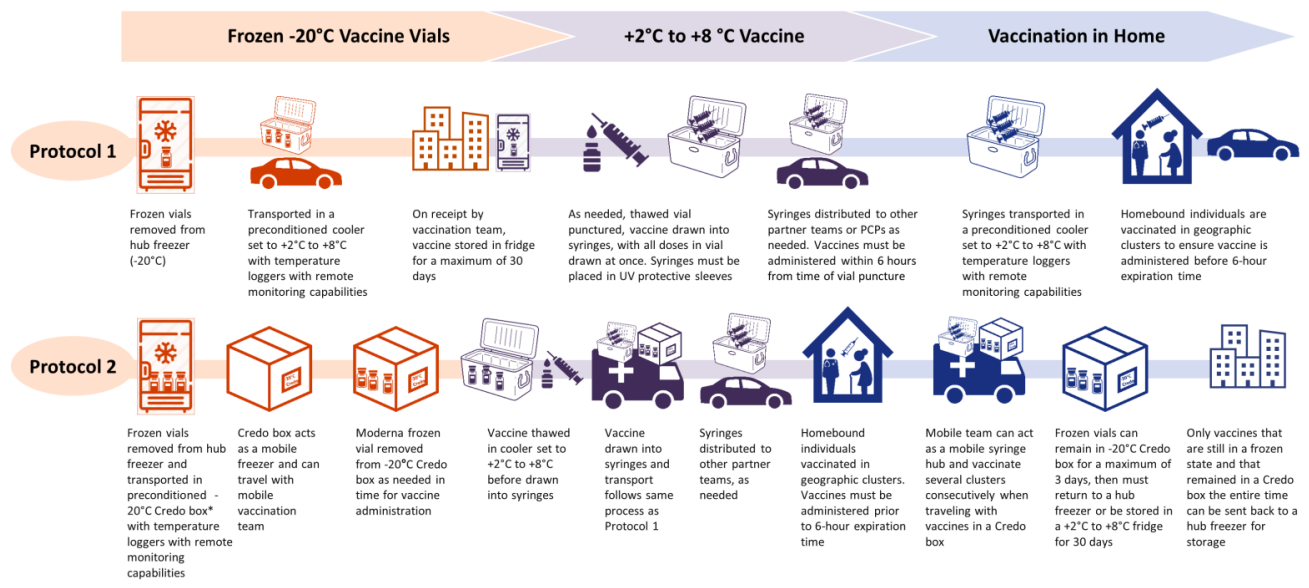

Figure 5. Map of Sample Vaccine Storage, Transport, and Handling Process for COVID-19 Vaccination of Homebound Individuals in Ontario

Protocols being rolled out for the City of Toronto, which can be used as sample protocols for the Province of Ontario. Protocol 1 involves transporting vials frozen-to-fridge with onward syringe transport. Protocol 2 involves transporting vials frozen-to-frozen in a Credo box, including transport of drawn syringes. *The hub freezer can either precondition Credo box TIC panels in $-80^{\circ} \mathrm{C}$ freezers or arrange for delivery of already preconditioned $-20^{\circ} \mathrm{C}$ Credo boxes. OHT, Ontario Health Team. PCP, primary care providers. TIC, thermal isolation chamber.

\section{Interpretation}

Many of Ontario's more than 75,000 homebound older adults receiving publicly funded home care services are currently or will soon be prioritized in Ontario's COVID-19 vaccination program, based on their advanced age, clinical risk factors, and residence in high SARS-CoV-2 incidence neighbourhoods. ${ }^{15}$ With 303,552 or $26.4 \%$ of Ontarians aged 75 years and above having neither received one dose of a COVID-19 vaccine nor booked an appointment to receive one, it is imperative that mobile in-home vaccination outreach is offered to the homebound population for whom this method of vaccination is a necessity and not a convenience, while prioritizing those in high-risk neighbourhoods to be vaccinated first. Additionally, there may be some homebound individuals that could visit COVID-19 vaccine clinics if supported with transportation. Ontario recently made a 3.7-million-dollar commitment to supporting individuals getting vaccinated who have transportationrelated barriers. ${ }^{16}$

The number of homebound individuals identified in our analysis is an underestimate of the total homebound Ontario population for several reasons. First, we only captured long-stay recipients of publicly funded home care, and there is likely another large population of homebound individuals who either rely solely on private home care providers or unpaid family caregivers. Second, the number of homebound individuals who relied on long-stay publicly funded home care services may have been lower than usual in the year 2020, as some individuals may have declined or cancelled services due to pandemic-related fears. To ensure that no homebound older adult is missed, jurisdictions like the City of Toronto will be activating a helpline to identify those requiring mobile in-home vaccination, including those without primary care providers. Other initiatives include the Toronto Public Library mobilizing its staff to reach out to older adult library card holders to support vaccine registration. ${ }^{17}$

We anticipate that the mobile in-home COVID-19 vaccination process, developed for the City of Toronto could be effectively replicated, and scaled up across the Province of Ontario. As many homebound individuals live with unpaid family or friend 
caregivers upon whom they are often reliant, and in some cases cannot be left unattended from, mobile in-home COVID-19 vaccination efforts should also include these caregivers. Finally, the approach to identifying homebound older adults and providing mobile in-home COVID-19 vaccination could also be used for other homebound populations such as younger individuals with disabilities.

\section{Methods Used for This Science Brief}

We included all Ontario adults aged 65 years and older who received at least one community-based long-stay home care assessment between January 1-December 31, 2020, using the Ontario Home Care Database (HCD). Long-stay home care assessments are completed every 6-12 months using the Resident Assessment Instrument - Home Care (RAI-HC) tool for the approximately $40 \%$ of Ontario home care recipients who are "long-stay", or expected to have care needs beyond 60 days. ${ }^{18-23}$ We next selected individuals who were homebound, based on a response of "no days" to RAI-HC variable G4b: "in the last 3 days, number of days went out of the house or building in which he/she resides".

We then reported the age and sex of these homebound individuals as well as their residence in one of Ontario's 34 PHUs. Finally, we stratified all homebound individuals within a PHU to a neighbourhood risk group based on the cumulative SARS-CoV-2 incidence in its forward sortation area (FSA; the first three characters of the building's postal code) from the beginning of the COVID-19 pandemic (January 23,2020 ) until January 16, 2021, with risk group 1 having the highest incidence and risk group 10 having the lowest incidence, by provincial decile; the methods for deriving these neighbourhood risk categories have been previously described. ${ }^{7}$

The City of Toronto COVID-19 vaccination strategy for homebound individuals was developed through a collaboration between Ontario Health Toronto Region, regional home care program leads for the City of Toronto, Toronto Paramedic Services, the University Health Network, and local primary care provider teams in partnership with Toronto Public Health. University Health Network Connected Care team provided backbone support that enabled daily steering committee meetings and the coordination of the work streams to develop, pilot and finalize ideal models for mobile in-home COVID-19 vaccination, using both primary care and paramedic vaccinators. University Health Network also leveraged its extensive experience supporting local mobile COVID-19 vaccination efforts in Toronto long-term care, retirement homes, and shelters and other congregate settings led by local primary care teams.

The protocols described in this brief for COVID-19 vaccine storage, transport, and handling were developed by the University Health Network vaccine hub and were piloted first with Ornge for the Operation Remote Immunity campaign, and then further piloted and refined for various local mobile vaccination initiatives, including the distribution of COVID-19 vaccine to other hubs across Ontario.

The development and operationalization of the protocols are aligned with the Ontario Ministry of Health guidance documents for the onward transport of COVID19 vaccines beyond the initial point of delivery and interim guidance for the transport of syringes containing COVID-19 vaccine for exceptional circumstances. ${ }^{10}$ SPRINT Senior Care House Calls' primary care provider team partnered in this process for the development of mobile COVID-19 vaccination models by leveraging pre-existing patient relationships, experience in home-based mass vaccination and expertise in home-based practices. Existing home visiting infrastructure, including 
scheduling, infection, prevention, and control (IPAC) protocols, and logistics, were adapted to meet the needs of a mass mobile COVID-19 vaccination program.

\section{Author Contributions}

NMS conceived the Science Brief and wrote the first draft. All authors revised the Science Brief critically for important intellectual content and approved the final version.

\section{References}

1. Jean-Jacques $M$, Bauchner $H$. Vaccine distribution-equity left behind? JAMA. 2021;325(9):829. https://doi.org/10.1001/jama.2021.1205

2. Ozawa S, Yemeke TT, Evans DR, Pallas SE, Wallace AS, Lee BY. Defining hard-toreach populations for vaccination. Vaccine. 2019;37(37):5525-5534. https:// doi.org/10.1016/j.vaccine.2019.06.081

3. Stall N, Nowaczynski M, Sinha SK. Back to the future: home-based primary care for older homebound Canadians: Part 1: where we are now. Can Fam Physician. 2013;59(3):237-240.

4. Qiu WQ, Dean M, Liu T, et al. Physical and mental health of homebound older adults: an overlooked population. J Am Geriatr Soc. 2010;58(12):2423-2428. https://doi.org/10.1111/j.1532-5415.2010.03161.x

5. Crawley M. Nearly 200,000 Ontarians age $80+$ have not signed up for a COVID19 vaccine. CBC. Published March 23, 2021. Accessed March 25, 2021. https:// www.cbc.ca/news/canada/toronto/covid-19-vaccine-ontario-bookingappointments-1.5958792

6. Government of Canada SC. Majority of Canadians intend to get the COVID-19 vaccine, September 2020. Statistics Canada. Published December 17, 2020. Accessed March 25, 2021. https://www150.statcan.gc.ca/n1/dailyquotidien/201217/dq201217c-eng.htm

7. Brown KA, Stall NM, Joh E, et al. A strategy for the mass distribution of COVID-19 vaccines in Ontario based on age and neighbourhood. Sci Briefs Ont COVID-19 Sci Advis Table. 2021;2(10). https://doi.org/10.47326/ocsat.2021.02.10.1.0

8. Ianzito C. Another COVID-19 challenge: vaccinating the homebound. AARP. Published March 4, 2021. https://www.aarp.org/health/conditions-treatments/ info-2021/covid-vaccines-for-homebound.html

9. City of Toronto. City of Toronto provides updated details of city COVID-19 vaccination program and collaboration with health sector partners. City of Toronto. Published March 1, 2021. https://www.toronto.ca/news/city-oftoronto-provides-updated-details-of-city-covid-19-vaccination-program-andcollaboration-with-health-sector-partners/

10. Ministry of Health and Long-Term Care. COVID-19 vaccine-relevant information and planning resources. Government of Ontario. Published March 24, 2021. https://www.health.gov.on.ca/en/pro/programs/publichealth/coronavirus/ covid19_vaccine.aspx

11. Centers for Disease Control and Prevention (CDC). Vaccinating homebound persons with COVID-19 vaccine. Centers for Disease Control and Prevention. Published March 2, 2021. https://www.cdc.gov/vaccines/covid-19/clinicalconsiderations/homebound-persons.html

12. Government of Canada. Recommendations on the use of COVID-19 vaccines. 
Canada.ca. Published March 16, 2021. Accessed March 31, 2021. https:// www.canada.ca/en/public-health/services/immunization/national-advisorycommittee-on-immunization-naci/recommendations-use-covid-19vaccines.html

13. Health Canada. Product Monograph Including Patient Medication Information: COVID-19 Vaccine Moderna. Government of Canada; 2021. Accessed March 31, 2021. https://covid-vaccine.canada.ca/info/pdf/covid-19-vaccine-moderna-pmen.pdf

14. Pelican BioThermal. Reusable passive thermal packaging. Published 2021. Accessed March 31, 2021. https://pelicanbiothermal.com/thermal-packaging/ reusable-parcel

15. Ministry of Health. COVID-19: Guidance for Priotization of Phase 2 Populations for COVID-19 Vaccination. Government of Ontario; 2021. https://www.afhto.ca/ sites/default/files/2021-03/Phase\%202\%20Prioritization\%20for\%20COVID-19\% 20Vaccination\%202021-03-22\%20FINAL\%20SHARE.pdf

16. Government of Ontario. Ontario helping people get their COVID-19 vaccination. Ontario.ca. Published March 23, 2021. Accessed March 31, 2021. https:// news.ontario.ca/en/release/60871/ontario-helping-people-get-their-covid-19vaccination

17. City of Toronto. City of Toronto continuing COVID-19 vaccine outreach to seniors. Toronto.ca. Published March 22, 2021. Accessed March 31, 2021. https://www.toronto.ca/news/city-of-toronto-continuing-covid-19-vaccineoutreach-to-seniors/

18. Poss J. Mind the Gap? Looking at Reassessment Patterns among Ontario Longstay Home Care Clients. Canadian InterRAI Conference; 2009.

19. Poss JW, Jutan NM, Hirdes JP, et al. A review of evidence on the reliability and validity of Minimum Data Set data. Healthc Manage Forum. 2008;21(1):33-39. https://doi.org/10.1016/S0840-4704(10)60127-5

20. Landi F, Tua E, Onder G, et al. Minimum data set for home care: a valid instrument to assess frail older people living in the community. Med Care. 2000;38(12):1184-1190. https://doi.org/10.1097/00005650-200012000-00005

21. Hirdes JP, Ljunggren G, Morris JN, et al. Reliability of the interRAl suite of assessment instruments: a 12-country study of an integrated health information system. BMC Health Serv Res. 2008;8(1):277. https://doi.org/10.1186/14726963-8-277

22. Health Quality Ontario. Home Care Indicator Review: Summary Report. Government of Ontario; 2017. Accessed December 14, 2020. https:// hqontario.ca/Portals/0/documents/system-performance/home-care-indicatorreview-report-2017.pdf

23. Ministry of Health and Long-Term Care. Presentation to the Premier's Council on Improving Healthcare \& Ending Hallway Medicine. Government of Ontario; 2018. 